or two years, but I do not believe the operation is justifiable, based on our knowledge of the location and behavior of gastric cancer. Cuneo's observations on the lymphatic currents were utilized by Hartman when he fixed the line of section in partial gastrectomy, and these facts have been accepted by our best surgeons.

In the radical operation for simple pyloric stenosis with hypertrophy, it has been suggested by Rodman that extensive resection beyond the lymph nodes may give better permanent results, because of the removal of pyloric ulcer and cancer areas, except in the dome and cardia. In cancer of the dome of the stomach or the cardia, total gastrectomy is a difficult and dangerous operation, with nearly 100 per cent. mortality, and if primarily successful will not give more continued relief than gastroenterostomy. In caricer of the body of the stomach a gastroplasty may be indicated if seen in the earlier stages. While the stomach is as abundantly supplied with blood vessels as any organ of the body, and anastomosis as perfect as in the vessels supplying the uterus, and in a degree similar, hemorrhage is easily controlled in radical operations by ligating the principal branches beyond the lines of incision. If the coronary branch of the gastric, the pyloric branch from the hepatic and the right and left gastroepiploica be ligated at the correct place, hemorrhage will not be profuse in radical operations at the pyloric end of the stomach extending up the lesser curvature nearly to the cardia. It must be remembered that the blood is derived from each of the three branches of the celiac axes-the gastric, splenic and hepatic. In partial gastrectomy I see no advantage in Mayo's curved incision with the convexity toward the dome, but prefer using the long straight forceps, and making a direct incision from the greater to the lesser curvature. This as successfully removes the infected area, and gives a broader and better stomach surface for jejunic attachment.

\section{THE PREVENTION OF TETANUS.}

\section{DANIEL N. EISENDRATH, A.B., M.D.}

Adjunct Professor of Surgery, College of Physicians and Surgeons: Attending Surgeon to Cook County Hospital.

\section{CIIICAGO.}

Much has been written during the past ten years concerning the symptoms and treatment of tetanus, and especially the frequency with which it followed all varieties of punctured wounds. Little, however, has been written as to definite methods for the prevention of this dread disease, and it has always seemed to me that this aspect has not been sufficiently dwelt on. We usually associate tetanus with Fourth of July wounds, but it is almost as frequent after punctured wounds received by rusty nails or pitchfork injurics as after blank cartridges. Why these three classes of injuries should be followed by tetanus can not be explained on any other ground than that in the case of blank cartridges the latter itself is free from tetanus, but that the hand or foot or face, which are the most frequent sites of infection, are more or less covered with street dirt containing tetanus bacilli, and that the blank-cartridge wound furnishes the ideal conditions for the development of the tetanus bacilli. These conditions are a more or less completely closed wound, containing a foreign body in the shape of a portion of the cartridge.

It has been shown experimentally that the pathogenicity of the tetanus bacillus is increased by irregular wounds where there is more or less necrosis, or in the presence of a hematoma, especially if a foreign body be present. In the case of rusty nails one does not have to seek far to explain the origin of tetanus after such wounds. It is not the rust on the nail, as has been generally thought, but the fact that the nail is covered more or less with dust and street dirt, and that the sole of the foot or the palm of the hands are, as in the case of a blank-cartridge wound, in none too clean a condition, being almost invariably. covered with dirt at the time of the reception of the injury. In the case of pitchfork wounds a somewhat similar origin can be demonstrated. It is well known at the present time that the tetanus bacillus is found under normal conditions in the intestine of horses, and that the spores are present in horsc dung, manured gardens, street dirt and poorly built wells. It does not require a great stretch of imagination to see how a pitchfork, either covered with street dirt or horse dung or garden earth, can carry into a punctured wound many tetanus bacilli. It has been amply shown that the blank cartridge itself does not contain tetanus bacilli. In the exhaustive article on "Fourth of July Casualties," published in The Journal of the American Medical Association, Aug. 29, 1903, five investigators are quoted who have examined 759 blank cartridges, bacteriologically, without finding tetanus bacilli in one.

My principal reason for writing this article has been to call attention to the fact that if a punctured wound which has been inoculated with tetanus bacilli be treated sufficiently early by methods which are based on the knowledge of the bacteriology of the disease, we can absolutely prevent the development of tetanus in every case. The tetanus bacillus, I need scarcely mention, is an anaërobic organism, and is surrounded by ideal conditions for its growth in punctured wounds. After the foreign body, whatever it may be, has penetrated the skin and underlying soft parts, the edges of the wound ontrance are rapidly closed by wound secretion, and we have a closed cavity, as I have been able to demonstrate on a number of occasions, devoid of oxygen, and an ideal place for the development of the tetanus bacillus. Add to this the necrosis and blood clots, and it requires only a short time before the symptoms of tetanus appear. When we consider that human beings, mice and guinea-pigs, are exquisitely sensitive to the toxins of tetanus, and that it requires only $1 / 100,000$ to $1 / 10,000,000$ of a cubic centimeter of tetano-toxin to kill a mouse, we can readily understand how a few tetanus bacilli can produce the necessary amount of havoc within a comparatively short space of time.

The period of incubation varies somewhat, according to the severity of the infection. According to Bruner, when the incubation period was one to five days, nine cases died and one recovered; when the period of incubation was five to ten days, eighteen died and eight recovered; when it was ten to twenty days, none died, and six recovered. From this we can deduce that the longer the period between the infliction of the injury and the appearance of the symptoms, the less severe the case. Many of the cases of tetanus which have responded to the tetanus antitoxin have belonged to the subacute variety.

In regard to the prospects of recovery after the development of tetanus, one needs only to bear in mind the large percentage of deaths, even after the use of antitoxin and medicinal remedies, such as morphin, bromids, chloral, etc. Ullrich has lately collected 19 cases, in which the Behring tetanus antitoxin was injected between the second and the fifth days. Of the nineteen, 
9 , or 47.3 per cent., recovered, and 10, 52.6 per cent., died. Including 53 cases treated during the same period of the appearance of the symptoms by other antitoxins, there are 72 cases, of which 39 (53.8 per cent.) were healed, and 33 died (45.5 per cent.) Twenty-three of these had an incubation period of ten days. Of these, 16 were healed ( 59.7 per cent.), which verifies what I have stated above, that the percentage of recoveries improves when the antitoxin is used late, because these are the milder cases; that is, those with longer periods of incubation. $70 \mathrm{f} 15$ cases collected by Kupnik, treated symptomatically with chloral, etc., 53 per cent. recovered. These were, in all probability, subacute cases:

In regard to the manner in which the tetanus toxin reaches the nerve-centers, there can no longer be any question that it is along the nerve trunks from the infected part, causing the characteristic symptoms through their toxic action on the nerve-centers of the pons and medulla, and then acting on the cranial nerves arising from this portion of the brain, thus explaining the early involvement of the fifth nerve. We thus see that the curative treatment of tetanus leaves much to be wished for, and we can only hope to reduce the high mortality of the disease through prophylactic treatment.

The technic which I am about to give can be summed up briefly as consisting of converting a closed wound filled with necrotic débris, blood clots and foreign bodies (blank-cartridge wads), into an open one, so that there can not be the least corner of it in which a tetanus bacillus finds its necessary conditions for growth. Before referring to my own work, let me call attention to a case which has been far more thoroughly worked out than any of those which $\mathrm{I}$ have to report, published in the Annals of Surgery of the present year, by J. B. Bain of the Massachusetts General Hospital. It is the first case in which tetanus bacilli were ever found before the development of symptoms of the disease, thus proving the theory that prophylactic treatment can actually be carried out, even though tetanus bacilli are in the wound, and can be demonstrated as such positively by bacteriologic tests. The case is as follows:

A boy of thirteen was shot in the palm of the right hand with a blank cartridge July 3, 1902. A punctured wornd was present, with blackened edges and surrounded by an area of powder-stained tissue. Every bit of blackened skin and tissue was removed; the wound thoroughly disinfected. All of the tissue, together with particles of the wad and powder grains, was placed in culture tubes and sent to the laboratory to be examined for tetanus bacilli. The latter were isolated in pure cultures. The patient made a prompt recovery without the development of any of the symptoms of tetanus.

A somewhat similar case has been reported lately by Ullrich. quoted above, in which a man's arm had been torn off at the shoulder joint. The patient was a diabetic, and the wound became infected with the pyocyaneus bacillus. No examination was made for tetanus bacilii. On the third day after the injury a prophylactic dose of antitetanic serum was given. No symptoms of tetanus appeared until two months after the injury, when he had twitchings in the face, pain on mastication, trismus, hyperesthesia, sweats, etc., these symptoms continuing for two months, and were interpretedas I believe to be perfectly justifiable-as having been tetanic.

During the past four years I have had occasion to treat eight cases of blank-cartridge and punctured wounds, with the direct view of preventing the development of tetanus. Of this number five were blank-cart- ridge wounds, three involving the fingers or palm of the hand, one the thigh, one the eyelid, and three cases were punctured wounds-one due to a pitchfork entering the ankle joint, and two rusty nail wounds penetrating the entire thickness of the foot from the sole of the foot to the dorsum. One of these cases described in detail will illustrate the treatment applied to all of them.

A boy 10 years of age was shot in the palm of the hand

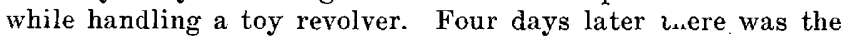
usual swelling in the palm of the hand and discoloration of the skin around the wound of entrance.

As in all of the above cases, where the wounds contained either blank-cartridge material or dirt which had been carried in by rusty nails or pitchforks, I would only consent to assume the responsibility in the case when the parents or the patient himself consented to the administration of an anesthetic in order to thoroughly cleanse the wound.

The cleansing was done in the present case in the following manner: An Esmarch constrictor was applied around the middle of the arm well above the point of infection, for the reason that one can only deal satisfactorily with any of the forms of infective wounds of the extremities when the tissues are bloodless, so that one can see the extent of the infection without constantly having the field of operation flooded with blood. After thorough disinfection of the entire forearm and hand by the usual methods, an incision was made directly over the palm of the hand and the entire tract of the blank cartridge followed. It extended from the middle of the palm of the hand to the base of the first phalanx of the middle finger. All of the connective tissue of the hand and the muscles lying between the metacarpal bones, as well as the tendon sheaths, were found to be encrusted with particles of powder, and the tract itself contained about a teaspoonful of the typical blackish-green pus which one encounters in this class of wounds. After wiping away the pus, a tissue forceps and scissors were employed to cut away every particle of powderstained tissue, as only in this manner can the wound be thoroughly cleansed. After this had been done, the edges of the wound having been held apart during the entire procedure by means of retractors, the skin along the edges of the tract, and especially around the wound of entrance, was excised, so that as far as the naked eye could determine, there were no blackish particles left in the wound. At the distal end of this suppurating tract a blank cartridge was found which showed a typical paper wad. Unfortunately cultures were not made at the time of the operation. The entire wound area was now swabbed with pure carbolic acid, followed immediately by the application of 96 per cent. alcohol, and packed with gauze, and a wet 1 to 1,000 salicylic acid dressing applied, and the hand was placed on a splint. No sutures were employed. The wound was allowed to heal by granulation. Immediately after putting the patient to bed, 20 cubic centimeters of tetanus antitoxin were injected to neutralize any of the tetanus toxin which might have been absorbed prior to the tirre of operation.

The after-treatment consisted in irrigating the wound daily with mild antiseptic solutions ( 1 to 1,000 salicylic acid and peroxid of hydrogen), and was then repacked. The patient made an uneventful recovery without showing any symptoms of tetanic intoxication.

A similar treatment was employed in all of the other cases, the first five not being given the prophylactic dose of tetanus antitoxin, but in spite of this making as prompt a recovery as the case that has been detailed.

\section{SUMMARY.}

The points which I desire to emphasize in the prophylactic treatment are the following:

1. Early and thorough exposure of every portion of the tract which has been infected by the blank cartridge 
or instrument which caused the punctured wound, retracting the edges, so that the disinfection and removal of the infected tissues can be done under the guidance of the eye.

2. Prophylactic injections of tetanus antitoxin to aid the organism in combating whatever toxins may have been absorbed prior to the time of operation.

3. This thorough disinfection can only be carried out under anesthesia, either local or general, and when the parts are rendered bloodless by the application of a constrictor.

4. The blank cartridge itself does not contain tetanus bacilli, but undoubtedly carries them in while penetrating the skin.

5. The instruments which cause punctured wounds either are covered as described above with tetanus bacilli or the manure of horses, etc., and are thus carried into the wound.

6. Our only hope in reducing the high mortality of tetanus is in a thorough application of such radical measures as have just been described.

\section{SUPRAPUBIC PROSTATECTOMY UNDER NI- TROUS OXID ANESTHESIA.* \\ JOSEPH WIENER, JR., A.B., M.D. \\ Adjunct Attending Surgeon, Mount Sinai Hospital. \\ NEW YORK CITY.}

That the removal of the prostate gland is the ideal surgical procedure in the treatment of prostatic hypertrophy is now very generally recognized. The chief contraindication to the performance of a prostatectomy up to the present time, according to the consensus of opinion, has been the danger resulting from the administration of ether or chloroform. Many surgeons have hesitated to offer some of their old prostatic cases the benefits of the operation on account of the presence of cardiac, nephritic or other lesions. It is in the hope of widening the field of usefulness of prostatectomy, and of extending the splendid benefits of the operation to patients who have heretofore had the advantage only of palliative operations, that this paper has been prepared. The cases which form the basis of the paper are, so far as I have been able to ascertain, the first cases of prostatectomy that have been performed without the use of ether or chloroform.

\section{CONTRAINDICATIONS.}

The chief contraindications to the performance of prostatectomy have until recently been the dangers resulting from first, the general anesthetic; second, hemorrhage; third, shock. By the use of laughing gas the great objection of a general anesthetic is at once removed. If, in addition, we do rapid work, then all three of the objections are removed. A prostatectomy done under nitrous oxid, in from eight to fifteen minutes, is not accompanied by any dangers from the anesthetic; the hemorrhage in those few minutes is very moderate and there is no shock. The patients are conscious even before the dressings have been applied. There is no vomiting; the pulse remains normal, and in a few minutes fluids are taken by mouth. Many. surgeons have held that a man unfit for general anesthesia is unfit for prostatectomy. In view of our experience with laughing gas, are we not justified in asserting that this view is erroneous? Is it not likewise erroneous to

- Read before the Harlem Medical Association of New York, March 2, 1904. hold that a man who can not take ether or chloroform should have a Bottini operation done? Is he not very much better off if he has a prostatectomy done under laughing gas? Diabetes is still considered by most surgeons an absolute contraindication to prostatectomy. So it probably is, if the operation takes forty-five minutes or longer, and if ether or chloroform is used. But if the suprapubic operation be done in ten minutes without ether or chloroform, then diabetes is no contraindication. 'Two of my cases were diabetics, and one of them had large amounts of glucose in the urine at the time of the operation and during the after-treatment. Yet both these cases recovered. A badly infected bladder is often given as a contraindication. In one of my cases (Case 5) there were daily chills as a result of a severe cystitis, and the urine contained a large amount of pus. After draining the bladder for ten days, the prostate was removed in toto, and the man made a splendid recovery. And that is just one of the advantages of the operation - that we can, in badly infected cases, first relieve the cystitis, and then, through the same opening, remove the prostate in a very few minutes. Again, we are told that old age is a bar to the removal of the prostate. The patient just referred to was 75 years old; another man was 82 years old. It is a curious fact that these two very old men made a more rapid recovery than the younger men. What, then, are the contraindications for the performance of a suprapubic prostatectomy under laughing gas? Candidly, I do not know. What I do know is that there are very few, even old diabetic cases with severe infections of the bladder, that can not be cured by the operation. This statement may seem extreme, but a careful study of the cases narrated below will, we believe, confirm its truth.

Many objections have been raised to the operation. Chief among them are severe hemorrhage, the infection of the raw surface left by the removal of the prostate, poor drainage and the difficulty of the operation. Chief among the objectors are those who do not perform the operation. As regards hemorrhage, I have never experienced a severe one, nor have I seen it at the hands of others. The infection of the raw surface at the base of the bladder we would expect, at first sight, to occur much oftener than it does. I do not know of a single case in which this complication was encountered. The use of urotropin and of bladder irrigations effectually prevents its occurrence. The opponents of the operation tell us that we are draining uphill. So we are. But by using syphon drainage, together with daily flushings of the bladder, we find that our drainage, from the very time of operation, is excellent. The entire wound is left open, and we never have any d fficulty in draining it. The same objection to uphill drainage might be raised in all our operations on the appendix and gall bladder. It would be just as valid as it is in regard to the bladder. The difficulty of performing the operation has been raised as an objection. We are told that great strength is required to shell out the prostate rapidly. The advantages of removing the gland in one piece are twofold: the hemorrhage is less and one is sure of having removed the entire gland. That a great deal of strength is generally required I can testify. The objection, however, does not apply to the operation, but to the operator.

\section{AGE OF PATIENTS.}

The youngest of my patients was 57 years old, and the oldest 82 vears. The average age was 69 years. 